\section{ce}

the

cardiovascular

response

to hypoxia

in wild-type

mice has

three distinct

temporal

phases

HYPERTENSION

\title{
Peripheral control of the systemic hypoxic response
}

The systemic vascular response to hypoxia is known to be regulated by the autonomic nervous system, whereas the role of the peripheral vasculature in this response is not clear. Now, Cowburn and colleagues have analysed the role of the tissue capillary bed underlying the skin in the systemic vascular response to hypoxia. "We used radio telemetry to noninvasively monitor blood pressure and heart rate in mice that have a keratinocyte-specific deficiency of hypoxia-inducible factor $1 a$ (HIF1a) or HIF $2 a$, or one of their respective target genes, nitric oxide synthase 2 (Nos2) or arginase 1 (Arg1)," explains senior author Randall Johnson.

Radio telemetry revealed that the cardiovascular response to hypoxia in wild-type mice has three distinct temporal phases: in the first ten minutes (immediate phase), both blood pressure and heart rate increase. The immediate phase is followed by a 36-hour period when both parameters fall below normoxic levels (acute phase), before the parameters return to normal levels by $\sim 48$ hours after hypoxia onset (chronic phase). The

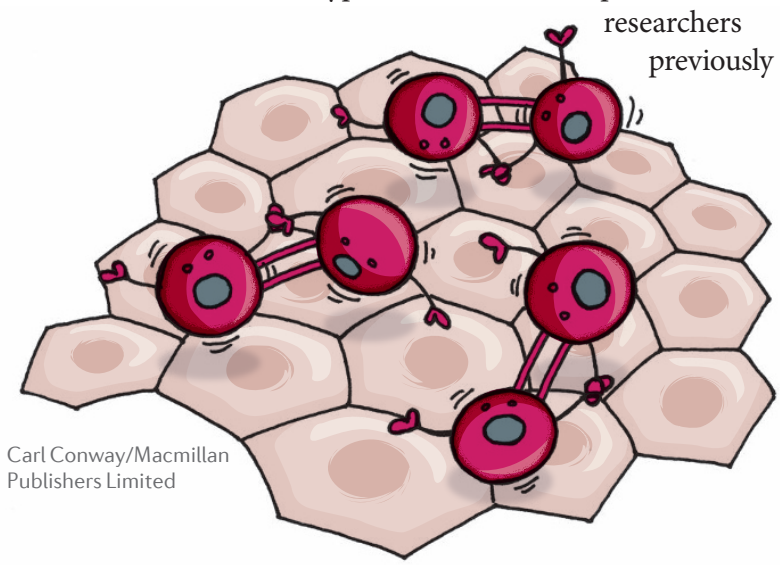

proposed a model in which HIF1 $\alpha-N O S 2$ and HIF2 $\alpha-A R G 1$ function in the homeostasis of nitric oxide (NO; an important signalling molecule that induces vasodilation) in the skin. Hypoxia induces an initial HIF1 $\alpha$-NOS2-mediated increase in NO levels that results in vasodilation, followed by activation of HIF $2 \alpha-A R G 1$, which indirectly reduces $\mathrm{NO}$ levels, resulting in vasoconstriction. Deletion of the hypoxia-response genes in the skin affects this triphasic systemic response: HIF1 $\alpha$ deficiency in mice delays the immediate increase in cardiovascular parameters in response to hypoxia, whereas HIF2 $\alpha$-deficient mice show a diminished immediate cardiovascular response to hypoxia. NOS2 or ARG1 deficiency only partially mirrors the effect of HIF1a or HIF2 $\alpha$ deficiency, respectively, on the systemic cardiovascular response to hypoxia, probably because of the compensatory activity of related NOS or arginase enzymes.

"These results demonstrate that the hypoxic response of the skin has an important role in cardiovascular adaptation to hypoxia, with HIF isoform-dependent functions in adaptive changes in cardiovascular parameters," concludes Johnson. "We hope to extend this study of peripheral response to other tissue types, in order to build a model of peripheral vascular resistance and response across the organism."

Grant Otto

ORIGINAL ARTICLE Cowburn, A. S., Macias, D. Summers, C., Chilvers, E. R. \& Johnson, R. S.

Cardiovascular adaptation to hypoxia and the role of peripheral resistance. eLife 6, e28755 (2017) 